\title{
Simulating the Impact of Heterogeneity on Stability and Effectiveness of International Environmental Agreements
}

\author{
Irina Bakalova ${ }^{\mathrm{a}, *}$, Johan Eyckmans ${ }^{\mathrm{a}}$ \\ ${ }^{a}$ Department of ECON-CEDON at KU Leuven, Warmoesberg 26, 1000 Brussels, Belgium
}

\begin{abstract}
This paper analyses in a systematic way the effect of benefit and cost parameter heterogeneity on the stability and effectiveness of International Environmental Agreements. Compared to existing literature, we consider a more general form of heterogeneity, different functional forms and alternative collective decision making processes in coalitions with and without transfers. Using systematic numerical simulations and novel visualization techniques, we show that transfers are crucial to overcome heterogeneity both in terms of stability and effectiveness. Without, or with limited transfers heterogeneous coalitions are often unstable and ineffective. More research on less than ideal transfer schemes and collective decision making rules is necessary to bridge the gap between theoretical models and the reality of international negotiations on transboundary pollution problems.
\end{abstract}

Keywords: $\mathrm{OR}$ in environment and climate change, international environmental agreements, simulation, asymmetry, game theory

JEL: C63, D7, F5, H41, Q5

\footnotetext{
${ }^{*}$ Corresponding author

Email addresses: irina.bakalova@kuleuven.be (Irina Bakalova), johan. eyckmans@kuleuven.be (Johan Eyckmans )

This research was funded by the KU Leuven internal research fund under contract OT/14/011.
} 


\section{Introduction}

Tackling effectively international environmental problems like global warming, depletion of the ozone layer, plastic waste in the oceans et cetera requires multilateral cooperation. Unilateral action by only one, or a few, countries cannot solve the problem if other polluting countries do not follow. But both from theoretical analysis and empirical observations, it is well known that global cooperation for solving international environmental problems is often very difficult to achieve. Because of the public good nature of emissions and waste reduction efforts, free riding by sovereign nation states often leads to insufficient provision of mitigation action (Barrett (2003)).

The problem of negotiating and implementing international environmental agreements (IEAs in the sequel) has been studied extensively in the environmental economics literature and has been reviewed by for example Finus (2001), Finus (2003) and Eyckmans (2012). The formation of so-called self-enforcing or stable IEAs is typically modelled using equilibrium concepts from non-cooperative game theory. In a seminal paper, Barrett (1994) considers welfare maximizing coalitions of homogeneous countries and shows that only small or ineffective IEAs can be stable in the sense that no insider wants to leave the agreement (internal stability) and no outsider wants to join the agreement (external stability). In other words, there seems to be a trade-off between broad participation and the mitigation ambition level. At about the same time, similar pessimistic conclusions about global cooperation for tacking international environmental problems were put forward by Carraro \& Siniscalco (1993) and Hoel (1991). Barrett (1997) and Diamantoudi \& Sartzetakis (2006) also showed that only small coalitions are stable, and McGinty (2007), Rubio \& Ulph (2007), and Pavlova \& de Zeeuw (2013) confirmed that participation can be increased only together with a reduction of the gains of cooperation. Real world observations seem to be in line with these theoretical results. For example, the 1997 Kyoto Protocol on the reduction of the emissions of six greenhouse gasses including $\mathrm{CO}_{2}$ can be interpreted as an example of a small IEA because only a limited subset of the polluting countries 
was willing to accept quantified emission reduction targets as was argued by Olmstead \& Stavins (2012). The recent 2015 Paris agreement on the other hand can be interpreted as an example of a broad but probably not very effective IEA. Many papers have shown that the combined mitigation pledges of the parties of the Paris agreement are most likely insufficient to keep global warming below $2^{\circ} \mathrm{C}$, see among others Fawcett et al. (2015) and Rogelj et al. (2016).

Assuming homogeneous countries is obviously restrictive and far from reality as was observed by McGinty (2007) and Eyckmans (2012) among others. For example, in the climate change context, countries differ strongly in emission reduction costs depending on the mix of energy sources, potential for renewable energy, energy efficiency etc. They differ also in terms of potential climate change damages in function of geographical and topographical conditions, adaptation possibilities etc. In addition, the heterogeneity in costs and damages can be correlated, positively or negatively. Intuitively speaking, assuming heterogeneity in costs of abatement and benefits from climate change mitigation can allow countries to exploit their differences and form a larger and more effective IEA. For example, if low (marginal) mitigation cost countries team up with high (marginal) mitigation benefit countries, they might be able to produce a substantial cooperation surplus. At the same time however, heterogeneity strongly increases the complexity of the analysis. First, there are different ways to model heterogeneity, and secondly, transfers among heterogeneous countries might enlarge the bargaining space for a stable IEA but they might also complicate the bargaining process.

In the theoretical environmental economics literature, there are several ways in which heterogeneity has been studied. In the following, we describe briefly how heterogeneity has been modeled so far. One way to introduce heterogeneity is to assume a limited amount of types of countries (typically two or four), that differ in their cost of abatement and damages from climate change (Barrett (1997, 2001); Fuentes-Albero \& Rubio (2010); Pavlova \& de Zeeuw (2013); Biancardi \& Villani (2010)). For example, Fuentes-Albero \& Rubio (2010) 
consider two types of countries and conclude that without transfers, heterogeneity has no relevant effects on the scope of international cooperation. Furthermore, even with transfers between coalition members, heterogeneity in abatement costs does not have significant impact on IEA formation. However, if countries differ in benefits of mitigation (hence, in damages from climate change) and use transfers, the level of cooperation increases with the degree of asymmetry. Hence, some forms of asymmetry seem to have a positive impact on stability of IEA.

Another way of analysing heterogeneous countries is more general and assumes that each country has its own unique characteristics (McGinty (2007); Colmer (2011); Finus \& McGinty (forthcoming)). Finus \& McGinty (forthcoming) consider a marginal change from symmetry to asymmetry and study its impact on the effectiveness of cooperating coalitions. The analysis leads to the conclusion that heterogeneity in both costs and benefits increases effectiveness of coalitions. Moreover, the authors show that heterogeneity in benefits improves stability of coalitions with transfers, while heterogeneity in abatement costs has no impact on coalition's stability. To sum up, the IEA literature started with models assuming homogeneous countries. Later heterogeneity was introduced in a specific way by considering a limited number of types of countries. The next step was to consider heterogeneity in a more general way, using a particular functional form for the heterogeneity. Recently, heterogeneity was considered without restrictions on its functional form, but only as a marginal difference from symmetry without considering the whole range of possible heterogeneity Finus \& McGinty (forthcoming). ${ }^{1}$

The overall purpose of this paper is to analyse the impact of heterogeneity in mitiga-

\footnotetext{
${ }^{1}$ There is still another strand of literature on heterogeneity and IEAs. This literature makes use of large scale numerical Integrated Assessment models. By investigating all possible subcoalitions of countries and regions, this literature tries to derive conclusions on what factors influence effectiveness and stability of IEAs, see Lessmann et al. (2015) for an example. Our approach is methodologically different. Lessmann et al. (2015) compare results of five different integrated assessment models in which regional characteristics are calibrated on real data. The resulting range of heterogeneity of parameters is therefore relatively limited. In contrast, we use a stylized theoretical simulation model that allows us to test our hypotheses on a much wider range of parameter heterogeneity.
} 
tion costs and benefits parameters on the effectiveness and stability of cooperation. We add to the existing literature by analysing the effect of heterogeneity in costs and benefit parameters on effectiveness and stability of coalitions in a very general way. We use two different functional forms for benefit and cost functions (linear benefits and quadratic costs versus quadratic benefits and costs), two different assumptions about the sequence of moves (simultaneous Nash versus Stackelberg equilibrium) and we allow for both positive and negative correlation between benefits and cost parameters. Also, we consider three alternative collective decision making procedures in coalitions, besides the usual assumption of joint coalition welfare maximization. In particular we consider voting by coalition members on a common emission tax rate to be applied within the coalition. Both standard majority voting and a minimum tax rate voting rule are included in our comparisons. Specific versions of each of these modelling assumptions have been studied separately in previous papers, but never their performance has been compared systematically. Also, never before the impact of general heterogeneity has been studied for each of the aforementioned modelling assumptions. For each model variant we solve the corresponding model analytically to derive individual abatement efforts, payoffs and coalition's surplus, stability and effectiveness measures. Then we set up a systematic numerical simulation exercise to generate a large simulated dataset to analyse the effect of heterogeneity on coalitions' effectiveness and stability. For this analysis we use novel graphical tools like heat maps to analyse the simulation results. Our results show that the widely held belief in the literature that heterogeneity in benefits is good for stability of IEAs seems to hold for a wider range of assumptions than used in the literature so far. However, the widely held belief that stability of IEAs would be rather insensitive to heterogeneity in mitigation costs does not seem robust for variations in functional form of the benefit function and game theoretic equilibrium concept.

The rest of the paper is organized as follows. We describe the model in Section 2, explain the numerical simulations in Section 3 and present results in Section 4. Section 5 
concludes.

\section{The Model}

Consider a stylized model of IEA formation with heterogeneous countries that emit a uniformly mixing pollutant ${ }^{2}$ and suffer from negative externalities from the total sum of emissions. $N=\{1, \ldots, n\}$ denotes the set of countries. Let $\bar{E}=\left\{\overline{e_{1}}, \ldots, \overline{e_{n}}\right\}$ be the vector of individual emissions in the situation when countries do not exert any effort to reduce their emissions, and $\bar{E}_{N}=\sum_{i \in N} \bar{e}_{i}$ is the total emissions in this case. Climate change is a typical example of such a problem because greenhouse gasses (GHGs) are uniformly and instantaneously mixing in the atmosphere. The increased concentration of GHGs causes the climate to change and can lead to damages through sea level rise, heat waves and drought, extreme weather and so on (see Cubasch et al. (2013)). Emissions can be reduced by abatement activities. We denote individual emission reduction by $q_{i} \in\left[0, \bar{e}_{i}\right]$ and assume it cannot exceed the initial emission level.

Reduction of GHG emissions mitigates climate change, and the avoided damages are considered in the model as benefits. Because GHGs are uniformly mixing, each individual country's benefits depend only on the total level of abatement $Q_{N}=\sum_{i \in N} q_{i}$. However, the intensity of benefits can be different across countries, reflecting for example differences in geographical characteristics (latitude, current climatic conditions, length of coastline etc. (see Burke et al. (2015); Carleton \& Hsiang (2016))), in level of development (rich countries typically have higher value at risk), adaptation possibilities etc. The relationship may be linear, as considered in Fuentes-Albero \& Rubio (2010); Pavlova \& de Zeeuw (2013), or quadratic, as in Diamantoudi \& Sartzetakis (2006); McGinty (2007); Weitzman (2014). We

\footnotetext{
${ }^{2}$ Other examples include emissions of chlorofluorocarbons (CFCs) and the depletion of the ozone layer, and, to some extend, disposal of plastics and the resulting pollution of the high seas.
} 
will present results for both specifications of the benefit function:

$$
\begin{aligned}
& B_{i}^{I}\left(Q_{N}\right)=b_{i} Q_{N} \\
& B_{i}^{I I}\left(Q_{N}\right)=b_{i}\left(Q_{N}-\frac{1}{2} Q_{N}^{2}\right)
\end{aligned}
$$

where $b_{i}$ denotes an individual benefit parameter, which indicates the combined net effect of size of the economy, vulnerability to climate change and adaptation possibilities. The linear benefits assumption implies that reaction functions of countries are orthogonal, which means that individual decisions about abatement effort do not depend on the abatement effort of other players. The quadratic benefits assumption implies negatively sloped reaction functions, which means that a player's optimal abatement is decreasing with the level of abatement of other players $^{3}$. In this case the outcome depends on the sequence of moves and we consider two types of interaction between the coalition and outsiders: simultaneous Nash equilibrium and Stackelberg equilibrium with the coalition acting as the leader and all singletons as followers.

At the same time, abatement activities are costly, and we assume that countries are characterized by different marginal abatement cost functions. Following Barrett (1994), Barrett (2001), McGinty (2007), Fuentes-Albero \& Rubio (2010) and many others in the IEA literature, we assume a quadratic specification of the cost function:

$$
C_{i}\left(q_{i}\right)=\frac{q_{i}^{2}}{2 z_{i}}
$$

where $z_{i}$ is country $i$ 's individual productivity in reducing emissions and $1 / z_{i}$ is the slope

\footnotetext{
${ }^{3}$ Some experiments show that alternative assumptions about the reaction of followers may hold. Heijden \& Moxnes (2003); Gächter \& Renner (2003) find that additional abatement efforts by a leader may serve as a good example for the followers, who increase their abatement efforts as well. However, the context of interpersonal interaction differs strongly from the context of interstate interaction. Therefore we should not immediately extrapolate the experimental results to problems in which countries interact .
} 
of an individual marginal abatement cost (MAC) function ${ }^{4}$. The higher is $z_{i}$, the more abatement can be done for the same costs. Typically, countries characterized by carbon intensive energy sources (like China) or by low energy efficiency (like Russia) are more productive in abatement then countries which already use a lot of renewable energy and are very energy efficient (like EU member-states). The reason for this is that energy efficient countries that use a lot of renewable energy can only reduce their emissions further by adopting expensive new technologies and by changes in consumers' behaviour. On the other hand, energy inefficient countries that rely mainly on fossil fuels can reduce their emissions by adopting existing and proven technologies at lower cost per ton of emissions abated. See Morris et al. (2012) for estimates of MAC functions using an applied general equilibrium simulation model.

Following Carraro \& Siniscalco (1993), Barrett (1994), IEA formation is modelled as a two-stage game. In the first stage countries decide whether to join a coalition or not, and in the second stage they choose abatement efforts given the coalition that has formed in the first stage. Regarding decision making within the coalition, usually only joint welfare optimization is considered, and therefore policy instruments are not explicitly specified. Welfare maximization is a theoretical benchmark solution for a coalition but it is not easily implementable in the real world. For this reason we consider two alternative decision making rules, based on voting by coalition members (all rules will be explained further in this Section). In order to analyse the different decision making rules, we consider a generic uniform carbon tax that applies to all members of the agreement. Each coalitional decision making rule boils down to a particular way of determining the uniform carbon tax rate. We do not consider redistribution of the national tax revenues. Hence, we assume that the tax revenue stays within the country and cannot be used for international transfers.

\footnotetext{
${ }^{4}$ In the IEA literature the following notation for abatement cost functions is common: $C_{i}\left(q_{i}\right)=c_{i} q_{i}^{2} / 2$, where $c_{i}$ is the slope of the marginal abatement cost function. The productivity parameter can however be easily converted to the cost parameter: $z_{i}=1 / c_{i}$.
} 
For the singletons we formulate maximization problem in terms of individual abatement in order to avoid confusion between tax rates of outsiders and coalition members. We do not focus on the internal policy instruments and assume that singletons can implement their individual abatement target by imposing a corresponding carbon tax or fixing emissions quantity.

The dependence of abatement in a country on the carbon tax rate can be modelled as the solution of a representative polluter's cost minimization problem $(4)^{5}$, which has the main feature that it equalizes marginal abatement costs to the carbon tax (5). Substituting with the quadratic cost function (3), we obtain a simple linear function that links the tax rate to the emissions abatement (5).

$$
\begin{gathered}
\min _{q_{i} \leq \bar{e}_{i}}\left(C_{i}\left(q_{i}\right)+t\left[\overline{e_{i}}-q_{i}\right]\right) \\
C_{i}^{\prime}\left(q_{i}\right)=\left(\frac{q_{i}^{2}}{2 z_{i}}\right)^{\prime}=\frac{q_{i}}{z_{i}}=t \quad \Rightarrow \quad q_{i}=C^{\prime}-1(t)=t z_{i} \in\left[0, \bar{e}_{i}\right]
\end{gathered}
$$

Cost efficient abatement is equal to the tax rate times the productivity parameter ${ }^{6}$. Hence more productive (i.e. lower marginal cost) countries will produce relatively more abatement effort for the same tax rate. Note that the tax rate should not exceed $\bar{e}_{i} / z_{i}$ in order for the abatement not to exceed $\bar{e}_{i}$. This restriction is explained in more detail in the online Appendix, Section A.3.

\footnotetext{
${ }^{5}$ The underlying idea is that there are many small symmetric polluters in a country such that the effect of a single polluter on the global emissions reduction is negligible and therefore individual benefits are not affected. That is distinct from the net benefit maximization problem of countries. Because of their size, countries can have a considerable impact on the global level of emissions reduction and hence on their own benefits.

${ }^{6}$ Note that the carbon tax scheme represents a cost efficiently implemented abatement target in general, which is driven by equalization of marginal abatement costs of polluters. Therefore, any common emission price instrument could do that job. For example, the coalition's abatement target may be implemented by means of an emission trading system, such that emission allowances are allocated with national auctions and all revenue stays within a country. Then $t$ is to be interpreted as the equilibrium price of emission permits in the market. Such a system is used since 2005 on a wide scale in the European Union. The Emission Trading Scheme ETS covers almost half of the EU's carbon emissions and the permit trading leads to a common and unique price of carbon throughout all affected sectors in all EU member states.
} 
Knowing individual abatement of coalition members, we obtain collective abatement of a coalition $Q_{S}$, where set $S=\{1, \ldots, s\}$ denotes the coalition members. We may express $Q_{S}$ as a function of the uniform tax rate $t$ and the sum of productivity parameters of coalition members, or signatories:

$$
Q_{S}(t)=\sum_{i \in S} q_{i}(t)=t \sum_{i \in S} z_{i}
$$

Total abatement of all countries $Q_{N}$ consists of the coalition's total abatement and the sum of all singletons (non-signatories) abatement levels, which, as we will see later in this section, also depend on $t$. Therefore $Q_{N}$ can be written as a function of $t$ and the productivity parameters:

$$
Q_{N}(t)=\sum_{i \in S} q_{i}(t)+\sum_{j \in N \backslash S} q_{j}(t)
$$

\section{Two-stage game}

The first stage of the game is the coalition formation stage. In this stage countries decide whether to join a coalition or to stay on their own. As in most of the literature on IEAs we assume for the first stage that only one coalition may be formed. In this stage all countries act simultaneously. Countries might be inclined to join a coalition because their marginal damages are fully taken into account when deciding on the coalition's mitigation

level. Joining the coalition will therefore result in higher emission reduction efforts by the coalition and lower climate change damages. This effect constitutes the main benefit of joining an agreement. At the same time however, coalition members have to adopt the uniform carbon tax which drives mitigation efforts. A higher carbon tax rate will lead to higher mitigation efforts and costs. The net effect of joining consists of the extra benefits (reduced climate change damages) minus the extra mitigation costs and might be positive or negative depending on the slopes of the benefit and cost functions, the size and composition of the coalition and the reaction by the other non-signatories.

In the second stage countries choose abatement levels given the coalition structure that 
has formed in the first stage. In the literature a distinction is made between simultaneous and sequential moves (Finus (2001)). We consider that players can either move simultaneously (Nash assumption), or that coalition members, who basically act collectively as a single player, behave as leaders in a Stackelberg equilibrium. We consider both types of equilibria as plausible as there is no empirical evidence available yet about which of both assumptions is more likely to prevail in the case of IEAs and international climate agreements in particular.

\section{Singletons' solution}

Singletons are assumed to maximize their individual net benefit function (8) for a level of abatement by other outsiders and by the coalition. As the abatement effort of the coalition is a function of the tax rate the coalition implements, the solution to this parametric problem is the optimal abatement level of a singleton $i$ as a function of the coalition tax rate $t$. In the main text we only state the maximisation problems of the players. The details of the solutions for the different modeling assumptions may be found in the online Appendix, Sections A.1-A.2.

$$
\max _{q_{i}} \pi_{i}=B_{i}(\underbrace{q_{i}+\bar{Q}_{N \backslash\{i\}}(t)}_{Q_{N}})-C_{i}\left(q_{i}\right) \quad \forall i \in N \backslash S
$$

\section{Coalition's solution: welfare maximization}

We consider three ways to determine abatement effort in a coalition. First, countries in a coalition choose a uniform carbon tax rate $t$ which maximizes total net benefits of coalition members:

$$
\max _{t} \sum_{i \in S} \pi_{i}=\sum_{i \in S}\left(B_{i}\left(Q_{N}(t)\right)-C_{i}\left(q_{i}(t)\right)\right)
$$

The welfare maximizing solution is a useful benchmark but it in reality it often seems too ambitious. First, we do not observe in the real world examples of agreements that 
explicitly aim at maximizing their joint welfare. As we referred to earlier, the pledges that countries made in the framework of the 2015 Paris climate agreement are insufficient to be in line with an emission path that keeps global warming below $2^{\circ} \mathrm{C}$, let alone $1.5^{\circ} \mathrm{C}$. Second, joint maximization often results in ambitiously high coalition targets, which makes it difficult to sustain cooperation. A less ambitious coalition may be more successful because of higher participation (Finus \& Maus (2008)). For these reasons we consider also two alternative scenarios for the coalition's decision making process based on voting procedures: the majority and minimal tax rules.

\section{Coalition's solution: majority tax voting rule}

Majority rule is one of the most popular decision rules in voting procedures. The idea has been used in the IEA literature (Hoel (1992); Weitzman (2014); McEvoy \& McGinty (2018)). If voters' preferences are defined over $t$ and are single-peaked, then majority voting produces an equilibrium which coincides with the peak preference of the median voter (Mueller (2003)). Preferences of countries are defined as $\pi_{i}\left(q_{i}(t)\right)$, which are quadratic in $t$ (and hence single-peaked) for all our considered functional forms. Details may be found in the online Appendix, Section A.4. Since preferences are single-peaked over $t$, the coalition tax is determined by the preferred tax of the median voter.

\section{Coalition's solution: minimal tax voting rule}

The minimal tax is a slightly different voting procedure that implements the lowest of the individually preferred taxes of the coalition members as the common tax. In the IEA literature it has been studied in Eyckmans (1999). This type of voting procedure is known to satisfy a voluntary participation constraint in the sense that every country will be at least as well off compared to a zero tax world. This is a necessary, but not sufficient, condition for countries to participate in an international environmental agreement. In particular, it is weaker than the internal stability requirement (11) that we will consider 


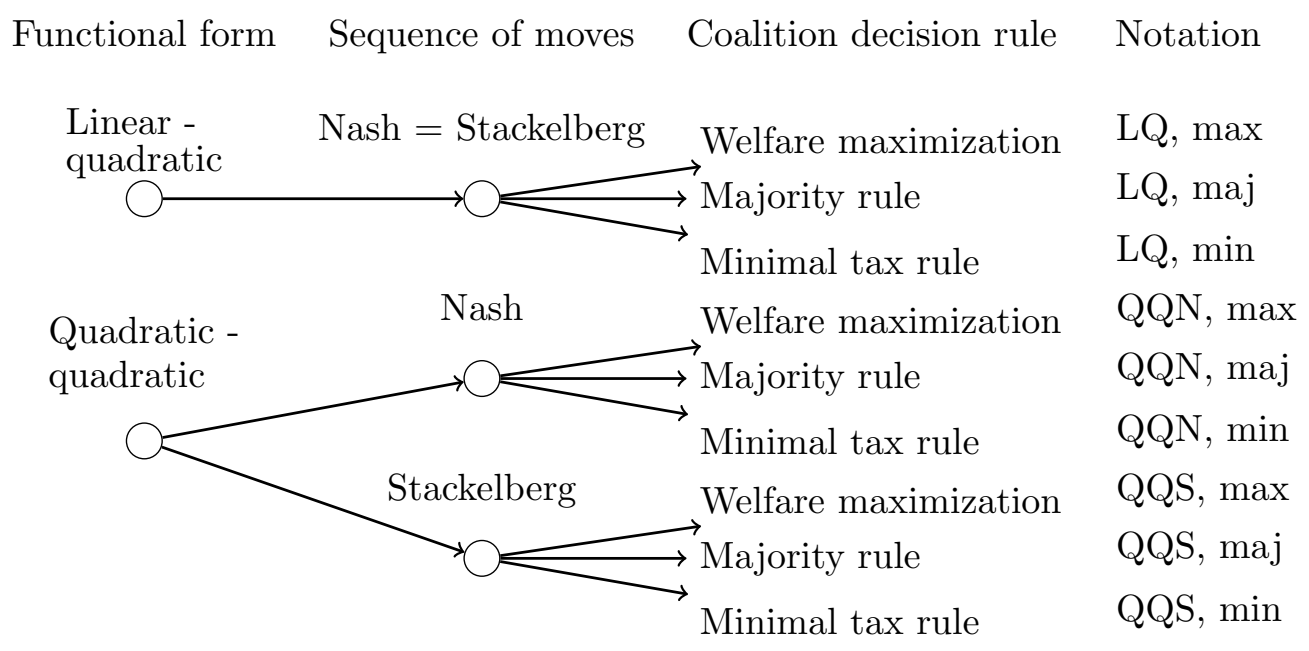

Table 1: Summary of all considered cases

later in this paper. Obviously, the minimal tax voting scheme will result in less ambitious emission reduction targets than the majority tax voting rule and therefore, it can have a higher potential to be stable because of the lower mitigation costs for participants. Table 1 summarizes all the cases which we consider in the analysis.

The game is solved by backward induction. First, we consider the maximization problems for singletons (8) and then the decision rule for the coalition members. Then, we solve the problems for an arbitrary coalition $S \subseteq N$, covering all possible outcomes ranging from no cooperation $(S=\emptyset$ or $|S|=1)$, over partial cooperation, to full cooperation $(S=N$, the grand coalition is formed). Analytical solutions for all functional forms and decision making rules are presented in the online Appendix, Sections A.1-A.2.

Given all possible coalitional solutions, we evaluate all possible cases using two criteria: effectiveness and stability.

\section{Effectiveness}

Global effectiveness of a coalition $S$ is measured as a closing-the-gap index (CGI). It shows 
the extent to which a given coalition "closes the gap" between total payoff of all $n$ countries in the no cooperation (defined as $\pi_{N}(\emptyset)$ ) and the first best scenario, i.e. welfare maximization in full cooperation $\left(\pi_{N}^{F B}(N)\right)$. The formula for CGI of a coalition $S$ is presented in (10), where total payoff of members and non-members of a coalition $S$ is defined as $\pi_{N}(S)$.

$$
C G I_{S} \equiv \frac{\pi_{N}(S)-\pi_{N}(\emptyset)}{\pi_{N}^{F B}(N)-\pi_{N}(\emptyset)}
$$

The denominator is always positive, because full cooperation always brings an improvement over no cooperation under the welfare maximization rule. However, the numerator is surely non-negative only in the linear benefits specification under welfare maximization, since there is no negative response by outsiders and welfare maximization always improves over the non-cooperative outcome. In the quadratic benefit function case, total payoff in no cooperation may be higher than under a coalition $S$, which implies a negative numerator ${ }^{7}$. Note that the maximum value of the CGI is 1, when total payoff under a coalition $S$ equals the first best scenario payoff.

\section{Stability}

For the game theoretic stability analysis we distinguish between scenarios with and without transfers among coalition members. Starting with the scenario without transfers, the condition for stability of an arbitrary coalition $S$ was provided by d'Aspremont et al. (1983). According to this definition, a coalition is stable if it is both internally and externally stable. A coalition is internally stable if no member is better off leaving the coalition (formulated in (11)), and it is externally stable if no outsider is better off joining the coalition

\footnotetext{
${ }^{7}$ For example, under the voting rules, it may happen that the equilibrium tax is very low such that some countries would be better off without cooperation. Also, in the quadratic benefit function specification, negative reactions by outsiders may slightly outweigh the additional abatement effort of coalition members, even under welfare maximization rule.
} 
(formulated in $(12))^{8}$.

$$
\begin{gathered}
\pi_{i}^{I N}(S) \geq \pi_{i}^{O U T}(S \backslash\{i\}) \quad \forall i \in S \\
\pi_{j}^{O U T}(S)>\pi_{j}^{I N}(S \cup\{j\}) \quad \forall j \in N \backslash S
\end{gathered}
$$

We also consider a scenario in which monetary transfers between coalition members are implemented by means of one of the schemes proposed by Eyckmans \& Finus (2004), McGinty (2007) and Weikard (2009). The underlying idea of all the schemes is that the coalition's joint payoff can be distributed among members in a way to pay each member at least its free-rider payoff. The free-rider payoff is the payoff that a player gets if he leaves the coalition and all the other members continue to cooperate, it can be formally written as $\pi_{i}^{O U T}(S \backslash\{i\})$ for $i \in S$. If the coalition's joint payoff is big enough to cover all the free-rider claims of its members, then a coalition is called Potentially Internally Stable (PIS) (Eyckmans \& Finus (2004)). The condition for PIS of a coalition $S$ is formulated in $(13)$.

$$
\pi_{S}(S)-\sum_{i \in S} \pi_{i}^{O U T}(S \backslash\{i\}) \equiv \nu^{S} \geq 0
$$

where $\pi_{S}(S)$ is the joint payoff of coalition $S, \nu^{S}$ denotes the surplus (or deficit) of coalition $S$ over the sum of free-rider payoffs of its members. A coalition's surplus is positive (or zero) for PIS coalitions and negative for unstable coalitions.

\section{Simulations}

Stability analysis in the quadratic benefits specification is not tractable analytically, since there is no closed-form expression for outside options of coalition members. To cope with this difficulty we apply numerical simulation techniques (using GAMS ${ }^{9}$ software) to

\footnotetext{
${ }^{8}$ If a player is indifferent (has equal payoffs in both options), we assume that he joins the coalition.

${ }^{9}$ See www.gams . com All programs used to produce this article are available from the authors upon request.
} 
analyse how heterogeneity affects internal, external and potential internal stability and effectiveness of coalitions. In the simulations we consider a set of 9 countries $^{10}$. We consider different values of the productivity $\left(z_{i}\right)$ and benefit parameters $\left(b_{i}\right)$ among coalition members. In general, different parameters values lead to different total levels $\sum z_{i}$ and $\sum b_{i}$, and these total levels of benefits and productivity have an additional impact on effectiveness and stability of coalitions, besides the effect of heterogeneity. In order to distinguish between these effects, we control total levels to be constant while simulating different distributions of parameters among coalition members. This assumption is commonly used in the literature analysing the effect of heterogeneity in IEAs (McGinty (2007); Finus \& McGinty (forthcoming)).

Different levels of parameter heterogeneity while keeping constant the total level is equivalent to different levels of concentration. Therefore it is appropriate to use concentration index as a measure of heterogeneity in our study. We choose the normalized Herfindahl index (14) to characterize distributions, because 1) it is designed to measure concentration levels and naturally suits for our problem; 2) it is neutral with respect to size, and therefore it enables to combine all coalition sizes to analyse general patterns. Normalized Herfindahl index varies between 0 and 1 for all coalition sizes $(s)$, moving from symmetry to strong asymmetry and concentration.

$$
H I_{b}=\frac{\sum_{i \in S}\left(\frac{b_{i}}{\sum_{i \in S} b_{i}}\right)^{2}-\frac{1}{s}}{1-1 / s} \in[0,1] ; \quad H I_{z}=\frac{\sum_{i \in S}\left(\frac{z_{i}}{\sum_{i \in S} z_{i}}\right)^{2}-\frac{1}{s}}{1-1 / s} \in[0,1]
$$

In the simulations we start with all players being symmetric $\left(b_{i}=z_{i}=1 \quad \forall i \in N\right)$. Then we consider all possible coalition sizes $s \in[2,9]$ and we apply a sequence of changes

\footnotetext{
${ }^{10}$ The number of countries does not affect the results significantly, because we have set up our study to analyse the effect of heterogeneity independent of size. We provide as an example simulation results for 25 countries in the online Appendix, section D.
} 
to generate different distributions of parameters $b$ and $z$, while keeping the parameters of outsiders unchanged. ${ }^{11}$ For each distribution of productivity and benefits parameters of coalition members we check whether a coalition is internally, externally and potentially internally stable, and calculate the closing-the-gap index. As a result, we have a simulated dataset, where each distribution of parameters is characterized by concentration indexes $H I_{b}$ and $H I_{z}$, and the corresponding game outcome is characterized by stability and effectiveness measures of a coalition. The procedure to change the parameter values of coalition members works as follows.

For each coalition size we start with symmetric players and then apply a sequence of equivalent changes of the productivity parameter. We randomly draw two coalition members and subtract a random number $\varepsilon$, drawn from a uniform distribution $U\left(0, z_{j}\right)$, from one player's productivity parameter $\left(z_{j}\right)$ and add it to the other one's $\left(z_{i}\right)$. In order to increase concentration, we always subtract from the lower parameter value:

$$
z_{i}^{\prime}=z_{i}+\varepsilon \quad \text { and } \quad z_{j}^{\prime}=z_{j}-\varepsilon, \quad \text { where } \quad z_{j} \leq z_{i}, \quad \varepsilon \sim U\left(0, z_{j}\right)
$$

In each step, once we have increased the concentration in productivity parameters ${ }^{12}$, we simulate the whole range of concentration in benefits with a similar procedure. We also control correlation between $b$ and $z$, in order to ensure that we have enough observations with both highly positive and highly negative correlation in the simulated dataset ${ }^{13}$. We

\footnotetext{
${ }^{11}$ We keep the parameters of outsiders unchanged for the sake of comparability of all considered specifications and scenarios. Heterogeneity of outsiders has an impact on their abatement level, which in turn influences the abatement target of a coalition in the quadratic benefits specification. In order to avoid this additional complication and to be able to compare the quadratic specification with the linear benefits specification, we design the simulation algorithm such that only coalition members are subject to equivalent parameter value redistributions.

${ }^{12}$ In order to have a nice coverage of parameter space and an equal spread over size classes, we impose an additional constraint on the heterogeneity level achieved at each step, full details may be found in the online Appendix, Section B.1. Combination of coalition sizes in grid cells is shown in the online Appendix, Section B.2.

${ }^{13}$ Since correlation has been shown to affect stability and effectiveness of IEAs (McGinty (2007)), we have to take into account this factor. We construct the dataset to have the whole range of correlation in each grid cell for the sake of generality of the analysis.
} 
start with positive correlation and control that all marginal changes increase the magnitude of concentration and correlation. Next we perform the same procedure for negative correlation.

In order to have a balanced dataset, we repeat the algorithm, but interchange the program loops: in the outer loop we increase $H I_{b}$, and in the inner loop we increase $H I_{z}$ for positive and negative correlation. Next, we repeat the described simulation algorithm for all coalition sizes, and finally we repeat the whole algorithm many times in order to have more observations in the dataset and increase the accuracy of the analysis. The simulation algorithm is shown in the online Appendix, Section B.1.

The results are discussed in Section 4.

\section{Results}

We consider both scenarios with and without transfers, two functional forms for the benefit function (linear and quadratic), two assumptions about the sequence of moves (simultaneous Nash and Stackelberg) and three different decision rules for a coalition to define its abatement target (joint welfare maximization, majority rule and minimal tax rule). We simulate about one million of possible distributions of mitigation benefits and abatement productivity parameters of coalition members. The analysis of the resulting dataset has been done in different ways, including multiple regression analysis. However, due to restrictions imposed by any specific functional form of econometric analysis and due to complications of interpretation (high number of interaction terms), we provide only a visual representation of the results in the paper.

\subsection{Stability without transfers}

We consider first the situation when there are no transfers between coalition members. We represent results using heat maps, which allow us to detect patterns in the simulation data. 
In the heat maps we simultaneously show the effects of heterogeneity in benefits parameters $\left(H I_{b}\right.$ - horizontal axis) and productivity parameters $\left(H I_{z}\right.$ - vertical axis). Moving left to the right corresponds with increasing heterogeneity in the benefits parameters while moving upwards corresponds to increasing heterogeneity in the abatement productivity parameters. To visualize the results, we divide the range of the Herfindahl Index into 10 intervals, both for the horizontal $\left(H I_{b}\right)$ and the vertical $\left(H I_{z}\right)$ axes, so overall we have 100 grid cells $^{14}$. Then we calculate the share of internally stable (IS) or/and externally stable (ES) coalitions in each grid cell. The share of IS or/and ES coalitions is depicted using color, increasing from blue to red. Rows in the combined figures correspond to different functional forms combined with the sequence of moves. The first row of graphs in the figures represents the linear-quadratic specification of the model. The second and third rows show the quadratic-quadratic specification, where the second row refers to the simultaneous move Nash equilibrium and the third row to the Stackelberg equilibrium. Columns refer to decision making procedures in the coalitions. The first column of heat maps shows results for the majority voting rule, the second column refers to the assumption of welfare maximization in coalition and the last column presents the minimal tax rule. The share of internally stable coalitions is depicted in Figure 1, the share of externally stable coalitions is shown in Figure 2, and the share of internally and externally stable coalitions is presented in Figure 3.

[Insert Figure 1 here]

Internal stability, welfare maximization

From Figure 1 we can observe that for the welfare maximization case (middle column of heat maps) the share of IS coalitions is extremely low as dark blue dominates in the heat maps. As could be expected, for the linear benefits specification and welfare max-

\footnotetext{
${ }^{14}$ It is possible to increase the "resolution" of the heat maps and have a finer picture. However, some noise may appear because of too small grid cells. To reduce noise, but without losses in essential information, we have decided to opt for 10 by 10 grid.
} 
imization assumption (top middle graph), the IS likelihood is zero or very close to zero confirming the well-known result by Barrett (1994). For the quadratic benefits specification and simultaneous move Nash equilibrium assumption, the probability of coalitions to be internally stable is always zero. Under the Stackelberg leadership assumption however, welfare maximization may lead to some internally stable coalitions as we can see from the heat map in the bottom middle graph. Up to one half of all coalitions are internally stable (corresponding to green in the heat map) if countries are close to symmetric in benefits (i.e. at the left side in the heat map) and maximize their joint payoff. Higher heterogeneity in benefits leads to lower share of IS coalitions. This shows that assumptions on functional forms and sequence of moves do matter for internal stability under heterogeneity. The result that under welfare optimization and in the absence of transfers heterogeneity cannot improve internal stability is in line with the literature (Fuentes-Albero \& Rubio (2010)).

\section{Internal stability, voting rules}

The majority rule voting procedure does not improve the situation, as we see from the first column of heat maps in the Figure 1. The share of IS coalitions is very low under all specifications. At the same time, if we consider the minimum tax rule (the last column of heat maps), we notice that the share of IS coalitions is considerably higher. We also see that it depends negatively on heterogeneity in benefits (moving left to right in the heat map, the color shifts from reddish over yellow and green towards blue). At the same time, internal stability seems to depend positively on heterogeneity in productivity (moving upwards in the heat map, the color shifts from blue towards green and orange). The share of IS coalitions is substantially higher in the Stackelberg equilibrium specification compared to other specifications. It even exceeds 0.9 if countries are symmetric in benefits (left side of the heat map) or extremely heterogeneous in productivity (top of the heat map). This can be explained by the fact that with high $H I_{b}$ and low $H I_{z}$ the voting equilibrium minimal tax goes to zero, and it is slightly positive with high $H I_{z}$ and low $H I_{b}$, which makes more coalitions with such characteristics internally stable. The dependence of a coalition tax 
on $H I_{b}$ and $H I_{z}$ is shown in the online Appendix, Section C. It is based on the fact that an individually preferred tax $\left(t_{i}^{*}\right)$ is increasing with $b_{i}$ and decreasing with $z_{i}$ (see online Appendix, Sections A.1, A.2). Intuitively, players with higher benefit parameters suffer more from climate change and would like to set more ambitious abatement goals. On the other hand, players with higher productivity would like to set relatively lower abatement target to avoid high abatement effort, because abatement is allocated cost efficiently among coalition members.

[Insert Figure 2 here]

\section{External stability}

In Figure 2 we see that in general share of externally stable coalitions is quite high, it exceeds 0.9 for any $H I_{b}$ and $H I_{z}$ in the linear benefits and quadratic benefits-Nash specifications under the welfare optimization assumption. For the majority and minimal tax rules we observe that share of ES coalitions depends negatively on heterogeneity in productivity and positively on heterogeneity in benefits. By and large, the picture for external stability is the mirror image of the picture for internal stability.

[Insert Figure 3 here]

\section{Both internal and external stability}

In Figure 3 we combine both conditions and show the share of stable coalitions without transfers. Overall we observe very little stability, the color blue dominates the heat maps. In particular under welfare maximization and majority rule, the share of stable coalitions is zero with few exceptions. Only for the minimal tax rule, we may say that coalitions consisting of countries that are heterogeneous in productivity have a relatively higher chance to be stable without transfers than coalitions that are more homogeneous in terms of mitigation productivity.

Overall, we can conclude that share of stable coalitions without transfers is very low. In the next subsection we consider transfers between coalition members and study the effect 
of heterogeneity of coalition members on its Potential Internal Stability.

\subsection{Stability with transfers}

At the 2009 Copenhagen climate policy summit, developed countries including the US, several EU member states, Canada, Australia, New Zealand etc., pledged to mobilise by the year 2020 at least 100 billion US\$ annually for climate finance. This pledge was reiterated on the eve of the 2015 Paris summit (Weikmans \& Roberts (2017)). Although the literature is skeptical about the governance of the transfer scheme, it shows that in the real world, transfers do play an important role in international climate policy making. Therefore we believe that analyzing stability and effectiveness of climate coalitions in a theoretical setting should pay attention also to scenarios that entail transfers among coalition members. With transfers, it does not make sense to consider the minimal and majority voting tax rules as before. Under the transfer scheme we use in this paper, the payoff to every coalition member is assumed to be proportional to the coalitional surplus. Hence, every member of the coalition has a strong incentive to strive for maximal joint welfare. Therefore, we only show results for the welfare maximization case.

[Insert Figure 4 here]

\section{Effect of heterogeneity in benefits}

From Figure 4, we see that heterogeneity in benefits from climate change mitigation has a positive impact on Potential Internal Stability (PIS) if coalition members maximize their joint welfare. Indeed, moving left to right in the heat maps, the color shifts from blueish towards reddish. Intuitively, this happens because with high $H I_{b}$, i.e. "concentration" of benefit parameters within a coalition, one or two members have very high $b_{i}$, whose benefits from climate change mitigation are huge and enough to cover free riding claims of others. Functional forms matter however for the share of PIS coalitions. Especially for the simultaneous move Nash equilibrium we observe a lot of blue, hence low shares of PIS coalitions $\left(H I_{b}\right.$ has to exceed 0.5 before the share of PIS coalitions attains 0.2 or more) 
compared to Stackelberg equilibrium or the linear benefit function specification. From the literature, it is known that the leader is better off and the followers worse off under the Stackelberg assumption compared to the sequential move Nash equilibrium (see Finus (2001), proposition 10.1). In other words, under the Stackelberg assumption, coalition members have higher payoffs, and at the same time lower outside options compared to the simultaneous move Nash equilibrium. This results in higher coalition surpluses, and therefore more PIS coalitions.

\section{Effect of heterogeneity in productivity}

We also notice that heterogeneity in productivity has a weak impact in the linear benefit function specification. Moving up or down in the heat maps does not bring us in other color areas in the heat map. This may be explained by cost-efficient allocation of abatement effort within a coalition. These findings confirm earlier results from the literature (McGinty (2007); Fuentes-Albero \& Rubio (2010); Pavlova \& de Zeeuw (2013); Finus \& McGinty (forthcoming)). For the Stackelberg equilibrium (and to lesser extent for the simultaneous move Nash equilibrium) however, we do observe that heterogeneity in productivity matters. In particular, higher levels of heterogeneity in productivity are associated with lower shares of PIS coalitions, especially for low to moderate levels of heterogeneity in benefits.

To sum up, monetary transfers scheme may substantially improve internal stability of welfare maximizing coalitions. The share of PIS coalitions is higher with higher heterogeneity of benefits parameters of coalition members. Also, the share of PIS coalitions is substantially higher when a coalition acts as a Stackelberg leader compared to simultaneous Nash equilibrium ${ }^{15}$.

\footnotetext{
${ }^{15}$ We do not present results for the effect of correlation, positive and negative, between benefit and productivity parameters because mostly, results are very similar. In one case however, we observed that countries with negatively correlated productivity and benefit parameters gain more from welfare maximizing cooperation with transfers compared to countries with positively correlated parameters. The intuition behind this observation is that in case of positive correlation (countries with high benefits at the same time
} 


\subsection{Effectiveness}

In this subsection we consider effectiveness of IEAs, measured by the closing-the-gap index (10). Cases with excessively high tax rates are not considered because of the natural restriction on emissions to be non-negative (see online Appendix, Section A.3). In Figure 5 we show the median value of CGI in each grid cell (similar as for stability, we divide the interval $[0,1]$ into 10 intervals when making a grid in the coordinates $\left.\left(H I_{b}, H I_{z}\right)\right)$. In some cases the CGI may be negative, i.e. the total payoff when a coalition $S$ is formed is less than in the non-cooperative scenario. We replace negative median CGI values with zero, since it is not rational for coalitions to choose for such outcomes. So, all negative and zero values are combined and depicted in dark blue in the heat map. The warmer the color is, the closer median CGI is to 1 , which refers to the first-best scenario.

[Insert Figure 5 here]

\section{Voting rules}

We observe from the figure that the minimal tax rule (third column) is associated very low effectiveness: The CGI is slightly positive only with countries that are almost symmetric in benefits in the linear benefits or simultaneous moves specifications. Considering both effectiveness and stability, we may say that under minimal tax rule, simultaneous moves Nash assumption and without transfers, coalitions with low $H I_{b}$ have a moderate CGI (0.2 to 0.3 ) and the share of stable coalitions exceeds 0.4. Majority rule brings about somewhat higher effectiveness, but as we have seen earlier in this section, this procedure does not lead to stable coalition without transfers.

\section{Welfare maximization}

Concerning the welfare maximization scenario, we may say that the median CGI is higher in the quadratic benefits specification, with higher $H I_{b}$ and lower $H I_{z}$. Also, in

have high productivity and vice versa), countries already do a lot of effort in no cooperation. In contrast, in case of negative correlation, countries benefit more from cooperation and use transfers to exploit their differences. 
simultaneous moves scenario it is higher than in the Stackelberg leadership scenario. This may be explained by the fact that the coalition tax in the Stackelberg leadership scenario is lower, since coalition members take into account the negative reaction of outsiders when deciding on their preferred tax level (see online Appendix, Section A.2).

\section{Conclusion}

In this paper we have analysed in a systematic way the effect of heterogeneity of coalition members' marginal benefit and mitigation cost parameters on the effectiveness and game theoretic stability of international environmental agreements. In that respect, our ambition was similar as several other papers in the IEA literature in the last years. We added to that literature by considering a very general type of heterogeneity that does not depend on a particular functional form of heterogeneity, on a limited number of country types or on only small parameter changes. Most of the literature so far has focused on the scenario in which coalition members are assumed to choose mitigation efforts in order to maximize joint coalitional welfare. In addition to that scenario, we have considered alternative decision making procedures within coalitions based on voting over a common uniform emissions tax rate. We also considered different functional forms for mitigation benefits (linear and quadratic) and assumptions on game theoretic equilibrium concepts for the second stage of the IEA formation game in which players choose abatement effort levels (simultaneous move Nash versus Stackelberg equilibrium). Methodologically we introduced novel heat maps to simultaneously depict how heterogeneity in benefits and cost parameters affect effectiveness (measured by a closing-the-gap index in global welfare) and game theoretic stability (measured by the share of coalitions that are internally, externally or potentially internally stable).

Our results confirm that when transfers are possible, heterogeneity in benefits goes together with higher stability (measured as PIS) and heterogeneity in productivity has little impact. We add to the literature by showing that this conclusion holds for a much 
wider range of assumptions than studied so far. However, our results also show that, without transfers and when coalition members use a minimal uniform tax procedure, more heterogeneity in benefits leads to lower stability and more heterogeneity in productivity leads to more stability. So far the literature has focussed on the optimistic assumption that coalitions choose mitigation efforts as to maximize their joint welfare. We add to the literature by showing that the assumption on the coalitions decision making rule is crucially important to understand the effect of parameter heterogeneity on stability of IEAs.

In terms of global effectiveness of IEAs, the uniform tax solutions (both the minimal and the majority voting tax rate solutions) typically produce insufficient mitigation compared to the global optimum and the coalition welfare maximization scenario as intuitively expected. We observe generally higher levels of effectiveness under the Nash compared to Stackelberg assumption. In addition, we show that under coalition welfare maximization rule heterogeneity in productivity is always bad for effectiveness, whatever the functional form or assumption on game theoretic equilibrium concept (Nash versus Stackelberg). Also heterogeneity in benefits is shown to be bad for effectiveness when we assume linear benefits. However, with quadratic benefits, more heterogeneity in benefits leads to higher effectiveness. Hence, our results show that the assumption regarding the functional forms of benefits crucially affects the effect of parameter heterogeneity on effectiveness, an insight that to our knowledge was not around in the literature so far.

Finally, we generally observe sharp trade-offs between stability and effectiveness of IEAs. Higher levels of stability often go hand in hand with lower levels of effectiveness for many scenarios. Only in a few specific cases, stability and effectiveness can be combined, be it on an intermediate level. This holds in particular for the welfare maximization scenario with transfers and, to a lesser extent, for the minimum uniform tax solution if heterogeneity in benefits is very low.

Which policy conclusion can be drawn from our results for real world negotiations on IEAs and on international climate agreements in particular? First, our results confirm that 
transfers enlarge the political bargaining space strongly and that heterogeneity is hampering neither effectiveness nor stability. Hence efforts should be continued to set up large scale transfer mechanisms in the Paris Agreement, not only via climate finance provisions but also via carbon pricing instruments with substantial redistribution of the revenues. Secondly, our results show that without, or with limited, transfers, agreements between widely differing countries in costs and benefits are unlikely to be stable and/or effective. In that case is seems more appropriate to focus on less ambitious targets among a smaller group of similar countries. In our opinion, after many years of intensive research on IEAs in the environmental economics literature, the impact of modelling assumptions on conclusions is still poorly understood. We hope our paper has offered an interesting contribution to the debate and we see several ways for future research. As we noted before, many papers assume joint welfare maximization as a coalition decision making rule. However, looking at the often slow progress and low level of emission reduction in international environmental agreements, we believe more analysis of alternative decision making rules is needed. An interesting direction of future research is to combine insights from game theory and political sciences on this topic. Also, most contributions in the literature neglect the dynamic nature of negotiations on international environmental problems. For example, the Paris agreement foresees regular revisions of the achievements and targets of the parties to the agreement. Incorporating this type of dynamic policy making is a challenging but relevant direction for further research. For each of these extensions, it is crucially important to account for heterogeneity of countries. We hope the results and approaches we offer in this paper can inspire this new research.

\section{References}

Barrett, S. (1994). Self-enforcing international environmental agreements. Oxford Economic Papers, 46, 878-894.

Barrett, S. (1997). Heterogeneous international environmental agreements. In C. Carraro, 
\& E. E. Mattei (Eds.), International Environmental Negotiations: Strategic Policy Issues (pp. 9-25). Edward Elgar UK.

Barrett, S. (2001). International cooperation for sale. European Economic Review, 45, $1835-1850$.

Barrett, S. (2003). Environment and Statecraft: The Strategy of Environmental TreatyMaking. Cheltenman, UK ; Northampton, MA: Oxford University Press.

Biancardi, M., \& Villani, G. (2010). International environmental agreements with asymmetric countries. Computational Economics, 36, 69-92.

Burke, M., Hsiang, S. M., \& Miguel, E. (2015). Global non-linear effect of temperature on economic production. Nature, 527, 235-239.

Carleton, T. A., \& Hsiang, S. M. (2016). Social and economic impacts of climate. Science, 353, 1112-1127.

Carraro, C., \& Siniscalco, D. (1993). Strategies for the international protection of the environment. Journal of Public Economics, 52, 309-328.

Colmer, J. (2011). Asymmetry, optimal transfers and international environmental agreements. GRI Working Papers Grantham Research Institute on Climate Change and the Environment.

Cubasch, U., Wuebbles, D., Chen, D., Facchini, M., Frame, D., Mahowald, N., \& Winther, J.-G. (2013). Introduction. In T. Stocker, D. Qin, G.-K. Plattner, M. Tignor, S. Allen, J. Boschung, A. Nauels, Y. Xia, V. Bex, \& P. Midgley (Eds.), Climate Change 2013: The Physical Science Basis. Contribution of Working Group I to the Fifth Assessment Report of the Intergovernmental Panel on Climate Change. Cambridge University Press, Cambridge, United Kingdom and New York, NY, USA.

d'Aspremont, C., Jacquemin, A., Gabszewicz, J. J., \& Weymark, J. A. (1983). On the Stability of Collusive Price Leadership. Canadian Journal of Economics, 16, 17-25.

Diamantoudi, E., \& Sartzetakis, E. S. (2006). Stable international environmental agreements: An analytical approach. Journal of public economic theory, 8, 247.

Eyckmans, J. (1999). Strategy proof uniform effort sharing schemes for transfrontier pollution problems. Environmental and Resource Economics, 14, 165-189.

Eyckmans, J. (2012). Review of applications of game theory to global climate agreements. Review of Business and Economic Literature, 57, 122-144.

Eyckmans, J., \& Finus, M. (2004). An Almost Ideal Sharing Scheme for Coalition Games with Externalities. Technical Report FEEM Working Paper No. 155.04. 
Fawcett, A., Iyer, G., Clarke, L., Edmonds, J., Hultman, N., McJeon, H., Rogelj, J., Schuler, R., Alsalam, J., Asrar, G., Creason, J., Jeong, M., McFarland, J., Mundra, A., \& Shi, W. (2015). Can paris pledges avert severe climate change? Science, 350, $1168-1169$.

Finus, M. (2001). Game theory and international environmental cooperation. New horizons in environmental economics. Cheltenman, UK ; Northampton, MA: Edward Elgar.

Finus, M. (2003). Stability and design of international environmental agreements: The case of transboundary pollution. In H. Folmer, \& T. Tietenberg (Eds.), The International Yearbook of Environmental and Resource Economics 2003/2004: a Survey of Current Issues (pp. 82-158). Cheltenham: Edward Elgar Publishing Ltd.

Finus, M., \& Maus, S. (2008). Modesty may pay! Journal of Public Economic Theory, $10,801-826$.

Finus, M., \& McGinty, M. (forthcoming). The anti-paradox of cooperation: Diversity pays! Journal of Economic Behavior and Organization, .

Fuentes-Albero, C., \& Rubio, S. J. (2010). Can international environmental cooperation be bought? European Journal of Operational Research, 202, 255-264.

Gächter, S., \& Renner, E. (2003). Leading by Example in the Presence of Free-Rider Incentives. Technical Report University of St. Gallen.

Heijden, E. v. d., \& Moxnes, E. (2003). The effect of leadership in a public bad experiment. Journal of Conflict Resolution, 47, 773-795.

Hoel, M. (1991). Global environmental problems: The effects of unilateral actions taken by one country. Journal of Environmental Economics and Management, 20, 55-70.

Hoel, M. (1992). International environment conventions: The case of uniform reductions of emissions. Environmental and Resource Economics, 2, 141-159.

Lessmann, K., Kornek, U., Bosetti, V., Dellink, R., Emmerling, J., Eyckmans, J., Nagashima, M., Weikard, H.-P., \& Yang, Z. (2015). The stability and effectiveness of climate coalitions: A comparative analysis of multiple integrated assessment models. Environmental and Resource Economics, 62, 811-836.

McEvoy, D. M., \& McGinty, M. (2018). Negotiating a uniform emissions tax in international environmental agreements. Journal of Environmental Economics and Management, 90, $217-231$.

McGinty, M. (2007). International environmental agreements among asymmetric nations. Oxford Economic Papers, 59, 45-62. 
Morris, J., Paltsev, S., \& Reilly, J. (2012). Marginal abatement costs and marginal welfare costs for greenhouse gas emissions reductions: results from the EPPA model. Environmental Modeling \& Assessment, 17, 325-336.

Mueller, D. (2003). Public Choice III. Cambridge University Press.

Olmstead, S. M., \& Stavins, R. N. (2012). Three key elements of a post-2012 international climate policy architecture. Review of Environmental Economics and Policy, 6, 65-85.

Pavlova, Y., \& de Zeeuw, A. (2013). Asymmetries in international environmental agreements. Environment and Development Economics, 18, 51-68.

Rogelj, J., den Elzen, M., Hohne, N., Fransen, T., Fekete, H., Winkler, H., Schaeffer, R., Sha, F., Riahi, K., \& Meinshausen, M. (2016). Paris agreement climate proposals need a boost to keep warming well below $2^{\circ} \mathrm{C}$. Nature, 534, 631-639.

Rubio, S. J., \& Ulph, A. (2007). An infinite-horizon model of dynamic membership of international environmental agreements. Journal of Environmental Economics and Management, 54, 296-310.

Weikard, H.-P. (2009). Cartel stability under an optimal sharing rule. The Manchester School, 77, 575-593.

Weikmans, R., \& Roberts, J. T. (2017). The international climate finance accounting muddle: is there hope on the horizon? Climate and Development, .

Weitzman, M. L. (2014). Can negotiating a uniform carbon price help to internalize the global warming externality? Journal of the Association of Environmental and Resource Economists, 1, 29-49. 


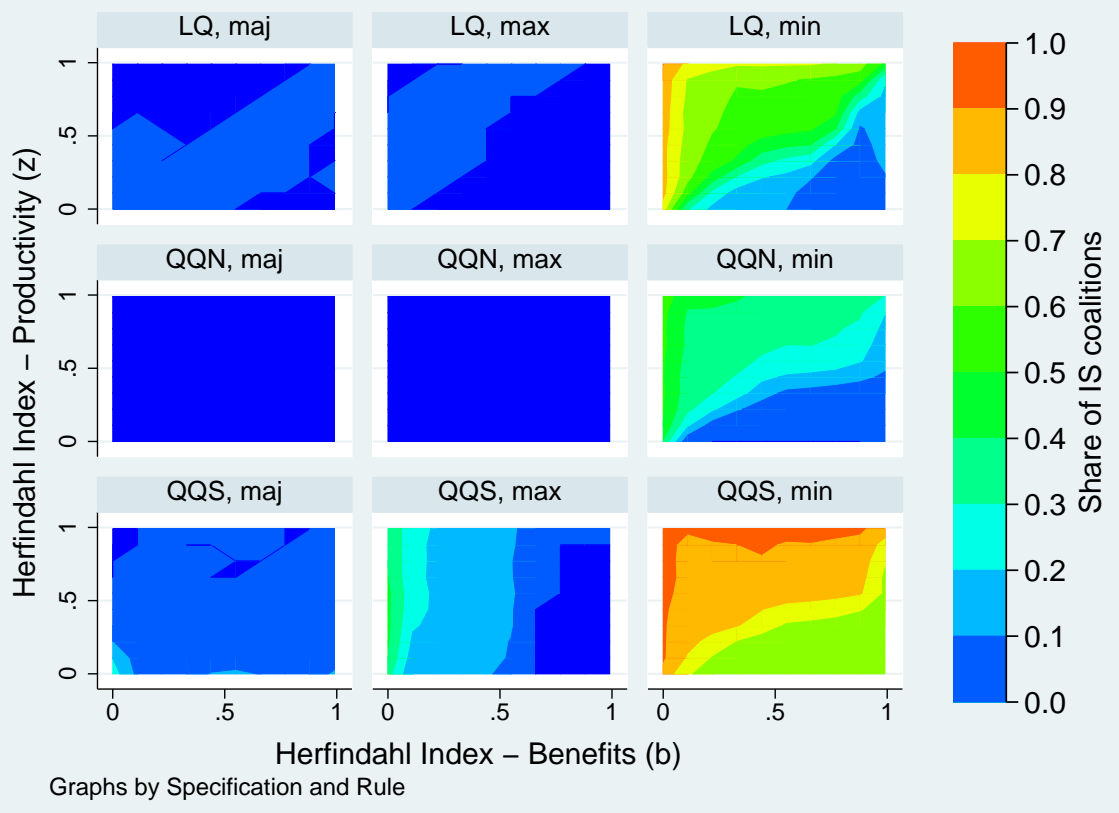

Figure 1: Internal Stability heat map. Specifications: LQ - linear benefits and quadratic costs functions; QQN - quadratic benefits and quadratic costs functions, simultaneous moves Nash equilibrium; QQS quadratic benefits and quadratic costs functions, Stackelberg leadership of a coalition. Decision rules in a coalition: maj - majority voting rule; max - joint welfare maximization; min - minimal tax rule. In color: share of internally stable coalitions in each grid cell.

\section{Figures}




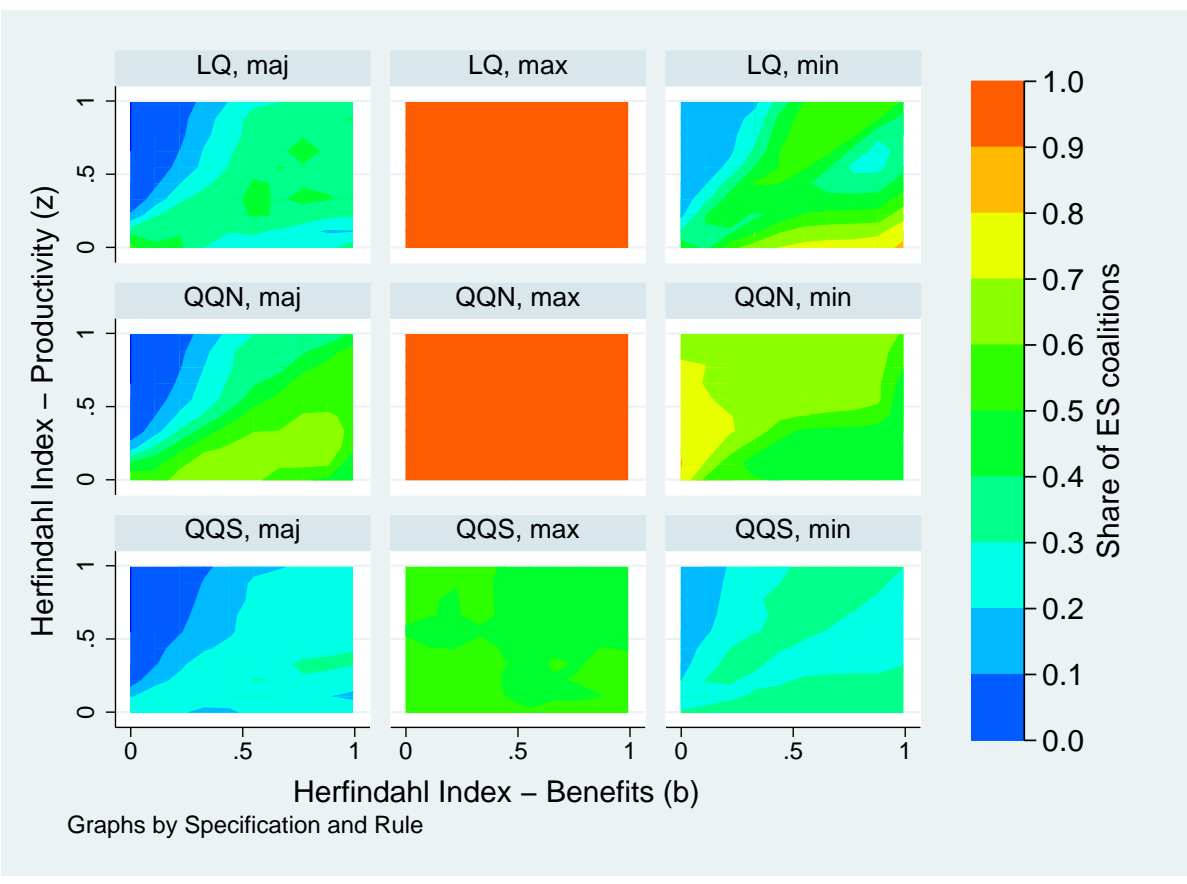

Figure 2: External Stability heat map. Specifications: LQ - linear benefits and quadratic costs functions; QQN - quadratic benefits and quadratic costs functions, simultaneous moves Nash equilibrium; QQS quadratic benefits and quadratic costs functions, Stackelberg leadership of a coalition. Decision rules in a coalition: maj - majority voting rule; max - joint welfare maximization; min - minimal tax rule. In color: share of externally stable coalitions in each grid cell. 


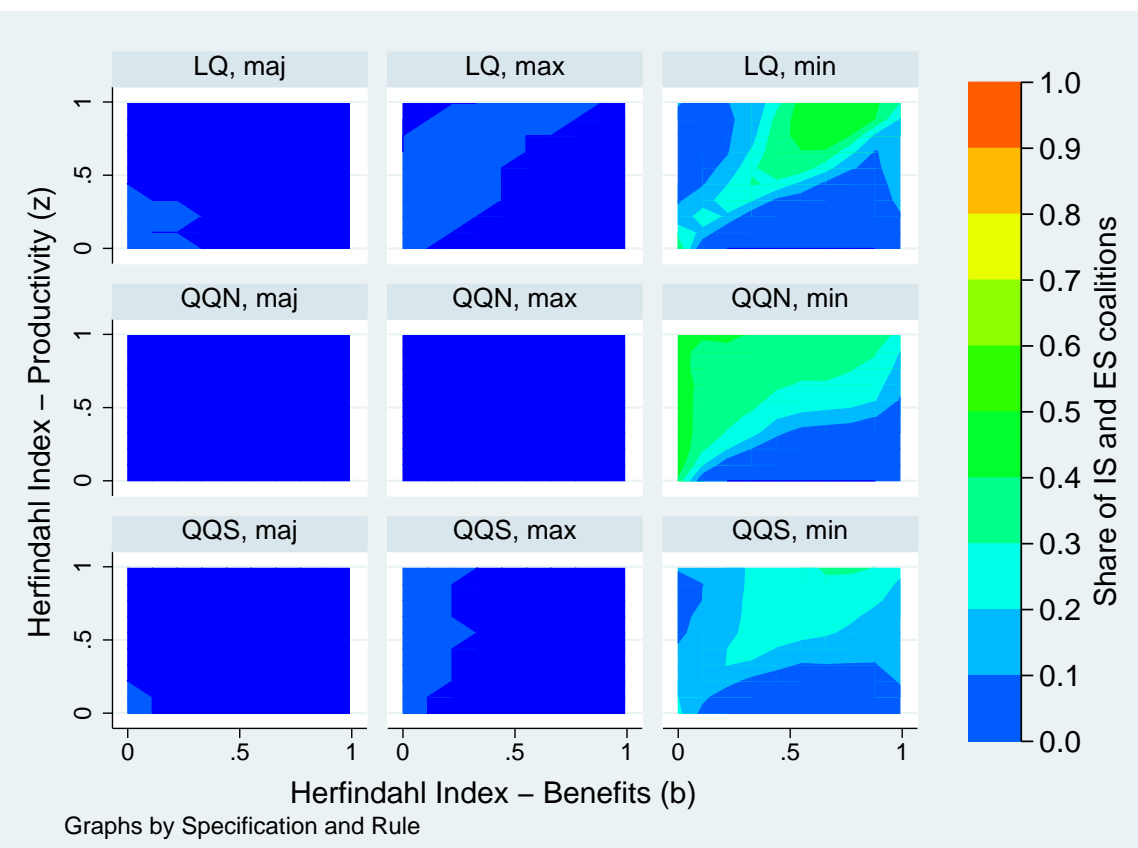

Figure 3: Internal and External stability heat map. Specifications: LQ - linear benefits and quadratic costs functions; QQN - quadratic benefits and quadratic costs functions, simultaneous moves Nash equilibrium; QQS - quadratic benefits and quadratic costs functions, Stackelberg leadership of a coalition. Decision rules in a coalition: maj - majority voting rule; max - joint welfare maximization; min - minimal tax rule. In color: share of both internally and externally stable coalitions in each grid cell. 


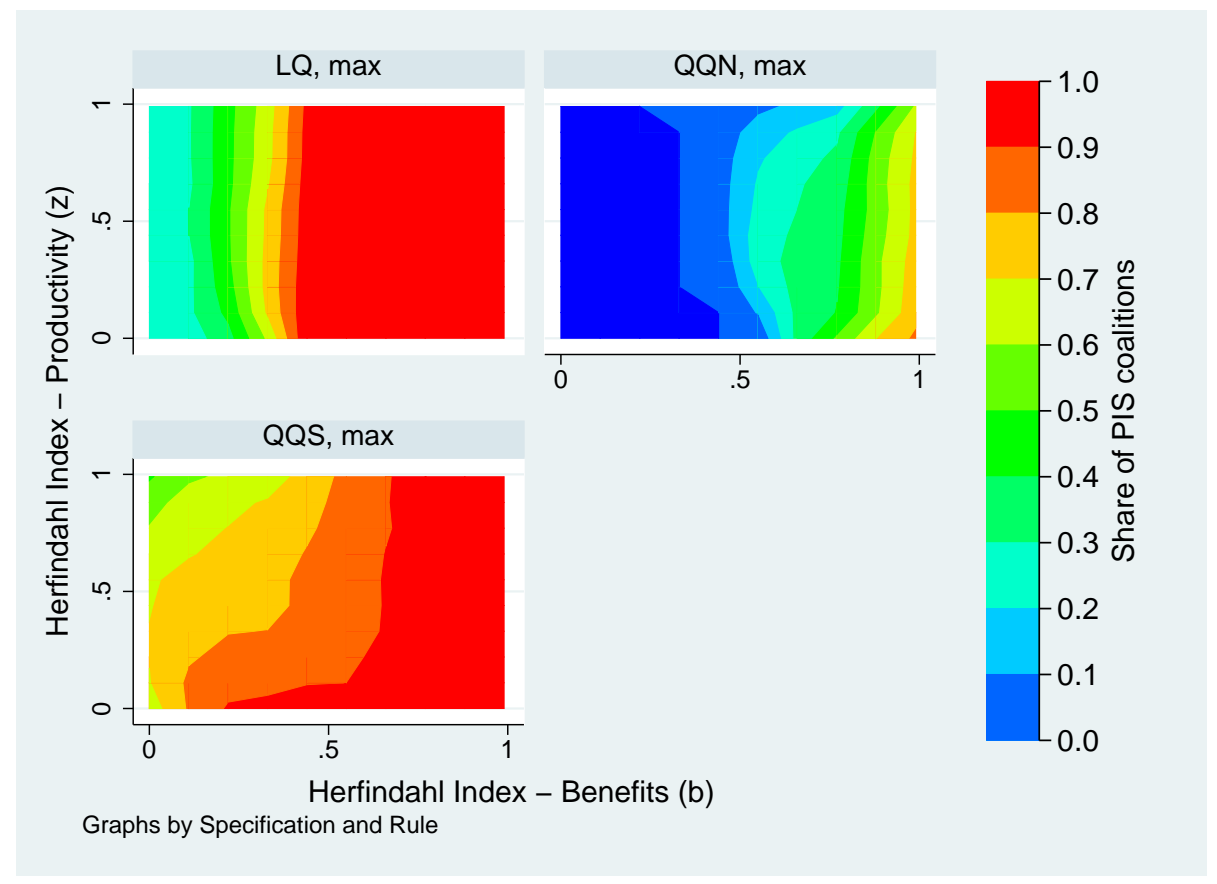

Figure 4: Potential Internal Stability heat map. Specifications: LQ - linear benefits and quadratic costs functions; QQN - quadratic benefits and quadratic costs functions, simultaneous moves Nash equilibrium; QQS - quadratic benefits and quadratic costs functions, Stackelberg leadership of a coalition. Decision rules in a coalition: $\max$ - joint welfare maximization. In color: share of potentially internally stable coalitions in each grid cell. 


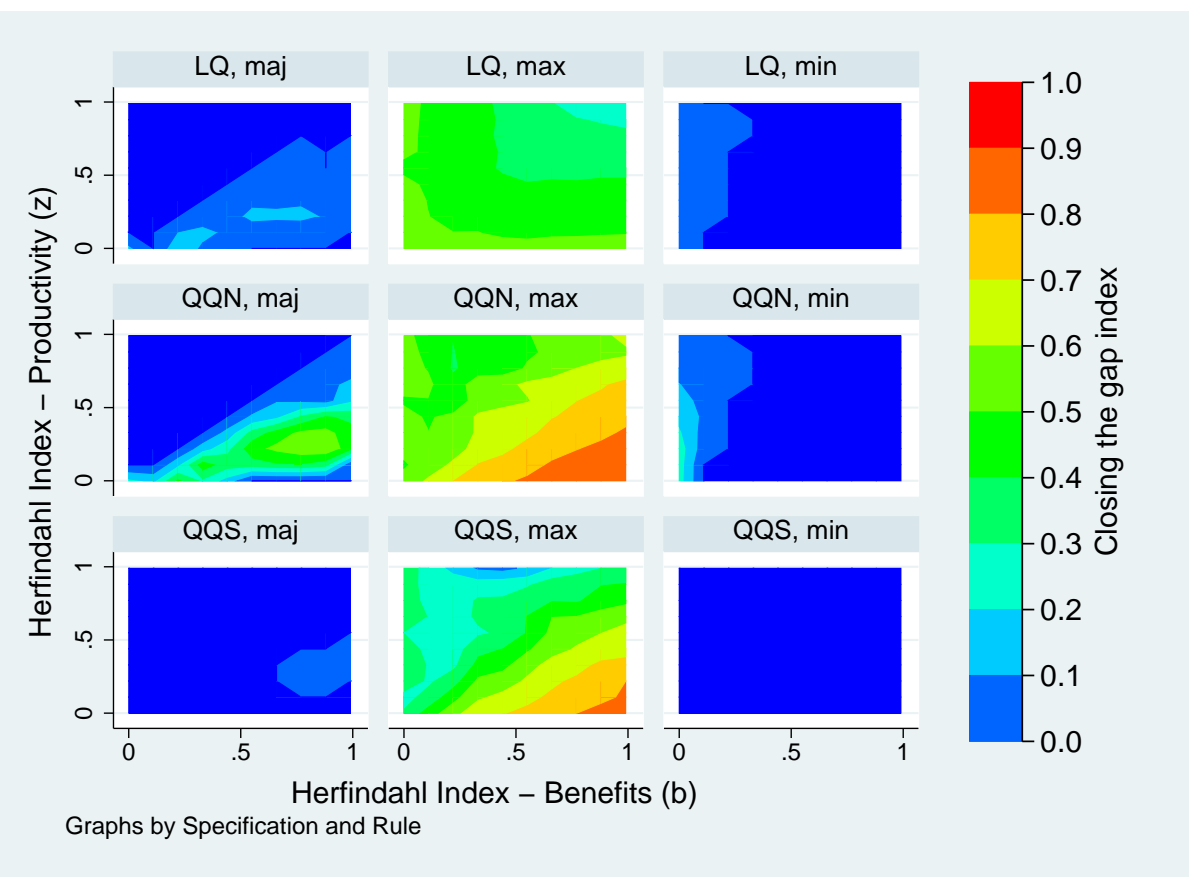

Figure 5: Closing-the-gap Index heat map. Specifications: LQ - linear benefits and quadratic costs functions; QQN - quadratic benefits and quadratic costs functions, simultaneous moves Nash equilibrium; QQS - quadratic benefits and quadratic costs functions, Stackelberg leadership of a coalition. Decision rules in a coalition: maj - majority voting rule; max - joint welfare maximization; min - minimal tax rule. In color: median value of closing-the-gap index each grid cell. 